\title{
Clarifying mammalian RISC assembly in vitro
}

\author{
Grace S Tan¹, Barry G Garchow ${ }^{1}$ X Xuhang Liu², David Metzler ${ }^{1}$ and Marianthi Kiriakidou ${ }^{{ }^{*}}$
}

\begin{abstract}
Background: Argonaute, the core component of the RNA induced silencing complex (RISC), binds to mature miRNAs and regulates gene expression at transcriptional or post-transcriptional level. We recently reported that Argonaute 2 (Ago2) also assembles into complexes with miRNA precursors (pre-miRNAs). These Ago2:pre-miRNA complexes are catalytically active in vitro and constitute non-canonical RISCs.

Results: The use of pre-miRNAs as guides by Ago2 bypasses Dicer activity and complicates in vitro RISC reconstitution. In this work, we characterized Ago2:pre-miRNA complexes and identified RNAs that are targeted by miRNAs but not their corresponding pre-miRNAs. Using these target RNAs we were able to recapitulate in vitro pre-miRNA processing and canonical RISC loading, and define the minimal factors required for these processes.

Conclusions: Our results indicate that Ago2 and Dicer are sufficient for processing and loading of miRNAs into RISC. Furthermore, our studies suggest that Ago2 binds primarily to the $5^{\prime}$ - and alternatively, to the $3^{\prime}$-end of select pre-miRNAs.
\end{abstract}

\section{Background}

MicroRNAs (miRNAs) are small ( 22 nucleotide) noncoding RNAs that associate with Argonaute proteins in ribonucleoprotein complexes (miRNPs or RISCs) [1-5]. MicroRNAs play diverse regulatory roles in development and physiological cellular functions [6-8]. In addition, miRNAs are involved in a wide spectrum of human diseases including cancer, cardiovascular and autoimmune inflammatory conditions [8-14].

Post-transcriptional nuclear processing of primary miRNA transcripts (pri-miRNAs) by the RNAse III enzyme Drosha and its RNA-binding partner, DiGeorge syndrome Critical Region gene 8 protein (DGCR8) [15-18], generates $\sim$ 65-75 nucleotide (nt) hairpin-structured miRNA precursors (pre-miRNAs). Pre-miRNAs are subsequently bound to the RAN-GTP protein Exportin-5 and are transported to the cytoplasm [19-22] where they undergo processing by the RNAse III enzyme Dicer into $\sim 22$ nt RNA duplexes [23,24]. TAR RNA binding protein (TRBP) facilitates Dicer processing of pre-miRNAs [25-28]. Ago proteins act as RNA chaperones facilitating unwinding of RNA duplexes and loading of single-stranded miRNAs into Ago complexes

\footnotetext{
* Correspondence: kiriakim@uphs.upenn.edu

'Department of Medicine, Division of Rheumatology, University of

Pennsylvania School of Medicine, Philadelphia, PA, USA

Full list of author information is available at the end of the article
}

$[29,30]$. The mechanisms involved in miRNA strand selection in mammals are poorly understood.

We previously reported that endogenous Ago2-premiRNA (pre-miRNPs) complexes are detected in cytoplasmic and nuclear extracts of human cells, and that pre-miRNPs are enriched in Dicer-null cells [31]. We showed that recombinant pre-miRNPs function as RISCs in vitro. This finding complicated our in vitro RISC reconstitution studies since recombinant Ago2 in complex with pre-miRNAs cleaves target RNAs bypassing the requirement for Dicer activity. In this study, we identified RNA targets that are not cleaved by Ago2:premiRNA complexes. We demonstrated that Dicer and Ago2 are sufficient for in vitro recapitulation of the cytoplasmic miRNP assembly and that TRBP is dispensable. We also showed that select Ago2:pre-miRNA complexes are active against 5'-as well as 3'-arm RNA targets, suggesting that Ago2 binds primarily to the 5'and alternatively, to the 3 '-end of select pre-miRNAs.

\section{Results}

Recombinant Ago2:pre-miR-24-1 complex directs cleavage of a $5^{\prime}$ - but not of a $3^{\prime}$-arm target

We previously reported that mammalian Ago2 directly binds to pre-miRNAs and forms active, non-canonical, RISC (pre-miRNPs) in vitro [31]. Ago2 can only use the hairpin-like pre-miRNAs as guides for target cleavage 
when spontaneous or Ago2-mediated dissociation of the hairpin arms permits target annealing to pre-miRNAs. Interestingly, two recent studies demonstrated RNA chaperone activity of human Ago proteins against RNA duplexes $[29,30]$.

Although the function and biological significance of endogenous pre-miRNPs is unclear, non-canonical RISC activity of pre-miRNPs complicates studies of RISC reconstitution. To bypass this alternative in vitro pathway, we first asked whether non-canonical RISC is also active against targets complementary to the 3'-arm of pre-miRNAs.

To test our hypothesis, we preloaded purified GSTAgo2 with pre-miR-24-1 in near stoichiometric amounts and added a 5 ' $-\mathrm{P}^{32}$-radiolabeled target complementary to either the 5'-or the 3'-arm of pre-miR-24-1. As expected, efficient 5 -arm target cleavage was detected in the absence of Dicer, whereas 3'-arm target cleavage was not observed (Figure 1). This suggests preferential activity of pre-miR-24-1:Ago2 complex against targets complementary to the proximal end of pre-miR-24-1.

\section{Ago2 and Dicer are sufficient for in vitro recapitulation of cytoplasmic steps of miRNA biogenesis}

Although in vitro reconstitution of mammalian RISC with recombinant Ago2, Dicer and TRBP has been previously reported, it is unclear whether in this study RISC was formed by Ago loaded with a Dicer-generated miRNA or unprocessed pre-miRNA [32]. Having demonstrated inactivity of Ago2-pre-miR-24-1 against a 3'-arm target, we were in a position to test RISC loading, by circumventing the non-canonical pathway. Ago2 was incubated with 5'-P-pre-miR-24-1 and Dicer, but not TRBP, then provided with a 5'-radiolabeled 3'-arm RNA target (Ta-3). Reaction products were analyzed by Urea-PAGE. Efficient cleavage of the radiolabeled Ta-3 was observed (Figure 2A), indicating TRBP-independent loading of the 3'-arm product of pre-miR-24-1 into Ago2. Inclusion of TRBP to the reconstitution reactions did not affect RISC activity (Figure 2A). These results indicate that Ago2 and Dicer alone were sufficient for canonical RISC loading in vitro.

In order to demonstrate that the 5 -arm product of pre-miR-24-1 was also loaded into Ago2, in addition to the 3'-arm product, the same reactions were repeated using 5'-radiolabeled pre-miR-24-1 and unlabeled RNA target. Labeled RNAs were analyzed by denaturing and native PAGE (Figure 2B and 2C). As expected, Dicer, but not Ago2 or TRBP, processed pre-miR-24-1 to $\sim 21$ nucleotide miR-24-1:miR-24-1* duplex, which was then dissociated by Ago2 and formed canonical RISC loaded with either miR-24-1 or miR-24-1* (Figure $2 \mathrm{C}$ and 2A). Although when these reactions were repeated with labeled pre-miR-24-1 and unlabeled target RNA, TRBP appeared to have a mild inhibitory effect on Dicer (Figure $2 \mathrm{~B}$ ), such effect was not observed when pre-miR-30 was used instead of pre-miR-24-1 (Additional File 1) or when RISC activity was tested (Figure 2A). Irrespective of regulatory activity, the presence of TRBP was also not essential for pre-miR-30a RISC loading reconstitution (Additional File 1). Together, our results indicate that recombinant Ago2 and Dicer are sufficient to recapitulate RISC assembly in vitro. We demonstrated unwinding of Dicer-generated miRNA duplexes in the presence of Ago2 and RISC loading with single-stranded miRNAs deriving either from the 3'-arm (as indicated by RISC activity shown in Figure 2A and Additional File $1 \mathrm{~A}$ ), or the 5'- arm of pre-miR-24-1 (as shown in Figure $2 \mathrm{~B}$ and $2 \mathrm{C}$ and Additional File $1 \mathrm{~B}$ and $1 \mathrm{C}$ ). TRBP is not essential for pre-miRNA processing by Dicer, unwinding of the RNA duplex or loading of miRNA or miRNA* into Ago2.

\section{RISC activity of select pre-miRNPs is guided against 5'- and 3 '-arm targets}

We tested several pre-miRNAs to demonstrate that a non-canonical Dicer-free Ago pre-miRNP is preferentially active against a 5'-arm target. Surprisingly, we observed 3'-arm target cleavage by select pre-miRNAguided RISCs. Pre-miR-24-1 and pre-miR-30a consistently demonstrated 5'- but not 3'-arm target cleavage, while pre-miR-138-2 and pre-miR-103-2 guided cleavage of targets complementary to both arms. In the case of pre-miR-138-2, the $17 \mathrm{nt} 5$ '- cleavage product of target Tb-5 (complementary to the 5'-arm of pre-miR-138-2), corresponds, as expected, to cleavage across the nucleotide in position 10 from the $5^{\prime}$-end of pre-miR-138-2 (Figure 3A). The 5'- product of the 3'-arm target Tb-3 $(16 \mathrm{nt})$ corresponds to cleavage across the nucleotide in position 10 from the 3'-end of pre-miR-138-2 (Figure $3 \mathrm{~A})$. An additional $18 \mathrm{nt}$ ' -product is seen, indicating cleavage across position 12 from the 3'-end. These findings suggest that, in vitro, in addition to binding to the 5'-phosphorylated end, recombinant Ago2 likely binds to the 3'-unphosphorylated end of pre-miR-138-2. Imprecise cleavage of the 3'-arm target resulting in two different size products suggests weak Ago2 binding to the unphosphorylated base of the 3'-end. An alternative explanation of these findings would be that Ago2 processes pre-miRNAs and is then loaded with guide RNAs deriving from their 3'- arm. However, we have previously [31] and currently demonstrated that such processing is not detected in vitro (Figure $2 \mathrm{~B}$ and Additional File 1B) at least with the conditions and premiRNAs we tested using purified, Dicer-free, recombinant Ago2, ruling out this alternative scenario.

Pre-miR-103-2, like pre-miR-138-2, directed cleavage of targets complementary to its 5'- and 3'-arms 


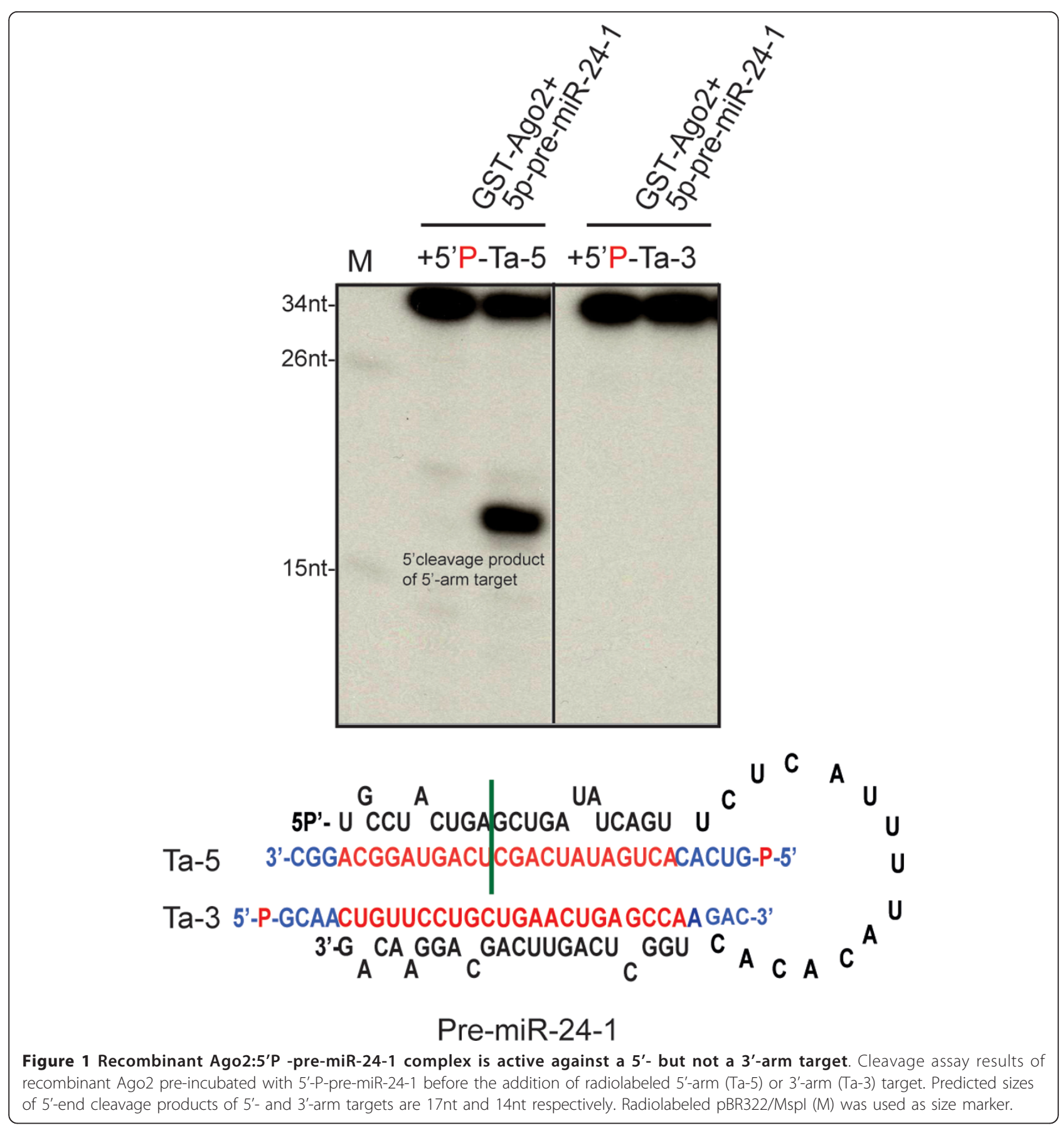

(Additional File 2). Imprecise cleavage of the 3'-arm target was noted again, as with pre-miR-138-2. In pre-miR103-2-guided cleavage, addition of recombinant TRBP was not crucial for the use of the 5'- or 3'- pre-miRNA arm by Ago2 (Additional File 2).

When Ago2 was loaded with a 73-nucleotide singlestranded, unstructured RNA, both 5' - and 3'- end targets were cleaved, although 3 '-end target cleavage was significantly less efficient (Figure 3B, and Additional File
3 for guide:target RNA alignment). This again suggests that Ago2 binds primarily, as expected, to the 5'-phosphorylated base of the guide RNA and can also bind to a small extent to the 3'- unphosphorylated base of the single-stranded guide RNA.

Endogenous Ago2-pre-miRNA complexes are detected in nuclear and cytoplasmic extracts of mammalian cells [31]. We have previously shown that in Dicer-null mouse embryonic fibroblasts (MEFs), endogenous Agos 


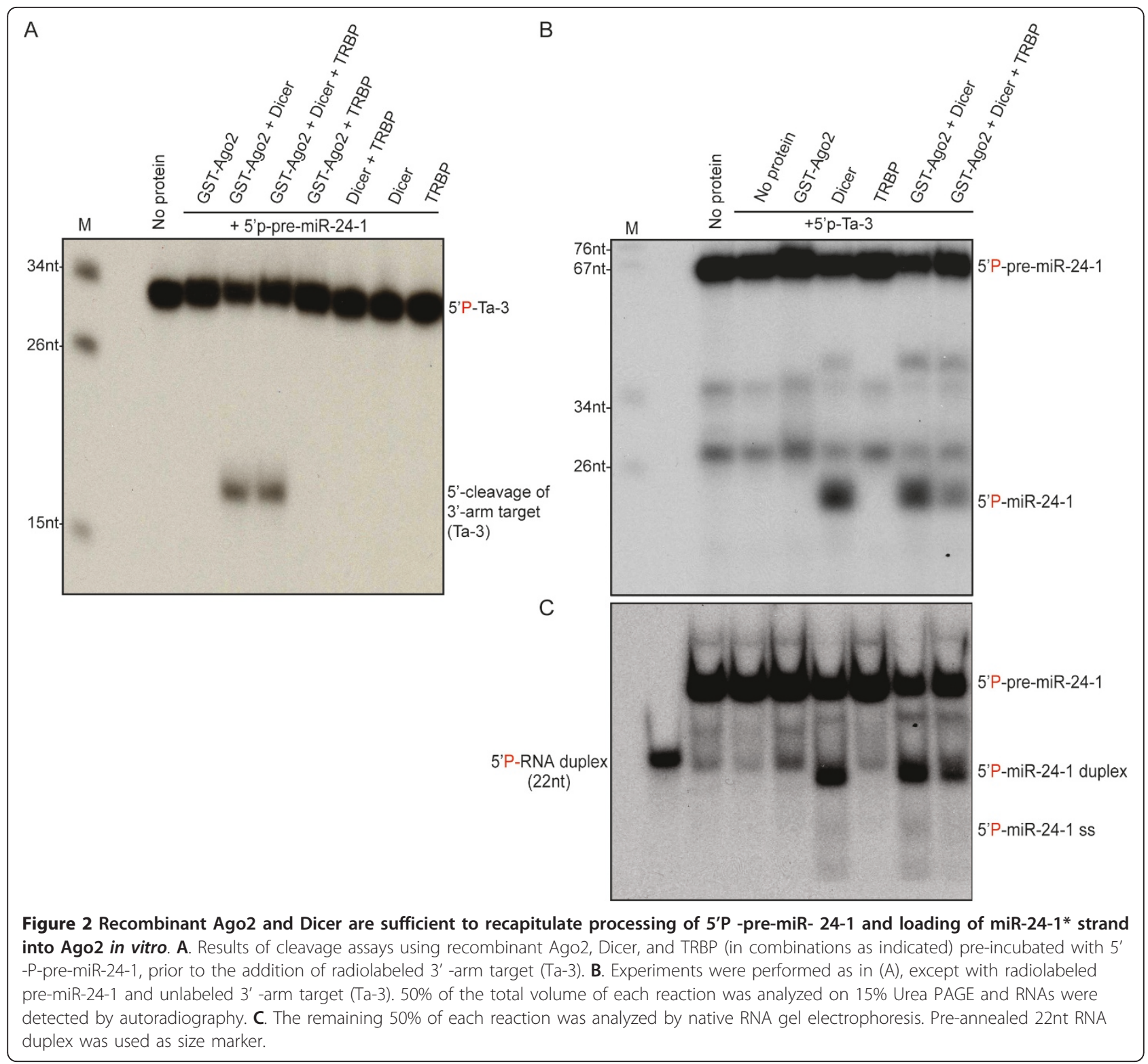

are loaded with unprocessed pre-miRNAs [31]. To test whether Ago2 binds to the 3'- end of endogenous premiRNAs, we immunoprecipitated Ago complexes from Dicer $^{-1-}$ MEFs using the 2A8 antibody[33] and performed in vitro RISC assays. In this inducible cell line a small amount of let-7a is detected (Figure 4B and[31]) even when Dicer is undetectable (Additional File 4). However, let-7a*, which normally originates from the 3'arm of pre-let-7a, is not detected in total RNA from Dicer $^{+/-}$or Dicer ${ }^{-/-}$MEFs (Figure 4C). To test the RISC activity of endogenous pre-let7-a RNPs, we provided RNA targets complementary to the 5'- and 3'-arm of pre-let-7a. In addition to the 5'-arm cleavage, a 3'-arm target cleavage product was observed, likely generated by RISC activity of pre-let-7a RNPs, rather than by canonical, let-7a* miRNPs (Figure 4A), suggesting that endogenous pre-let-7a associates to Ago2 via either its 5'-phosphorylated or its 3'- unphosphorylated end.

\section{Discussion}

Most endogenous pre-miRNAs are normally processed by Dicer and consequently functional miRNPs are more abundant than pre-miRNPs [31]. We have previously shown that canonical pre-miRNAs associate with Ago2 and that in vitro, Dicer-free Ago2 does not process premiRNAs into mature miRNAs or any intermediate products [31]. It was subsequently shown that Ago2 binds to pre-miR-451 and mediates its processing via a noncanonical, Dicer-independent pathway [34,35]. Unlike most mammalian miRNAs, pre-miR-451 has a unique 


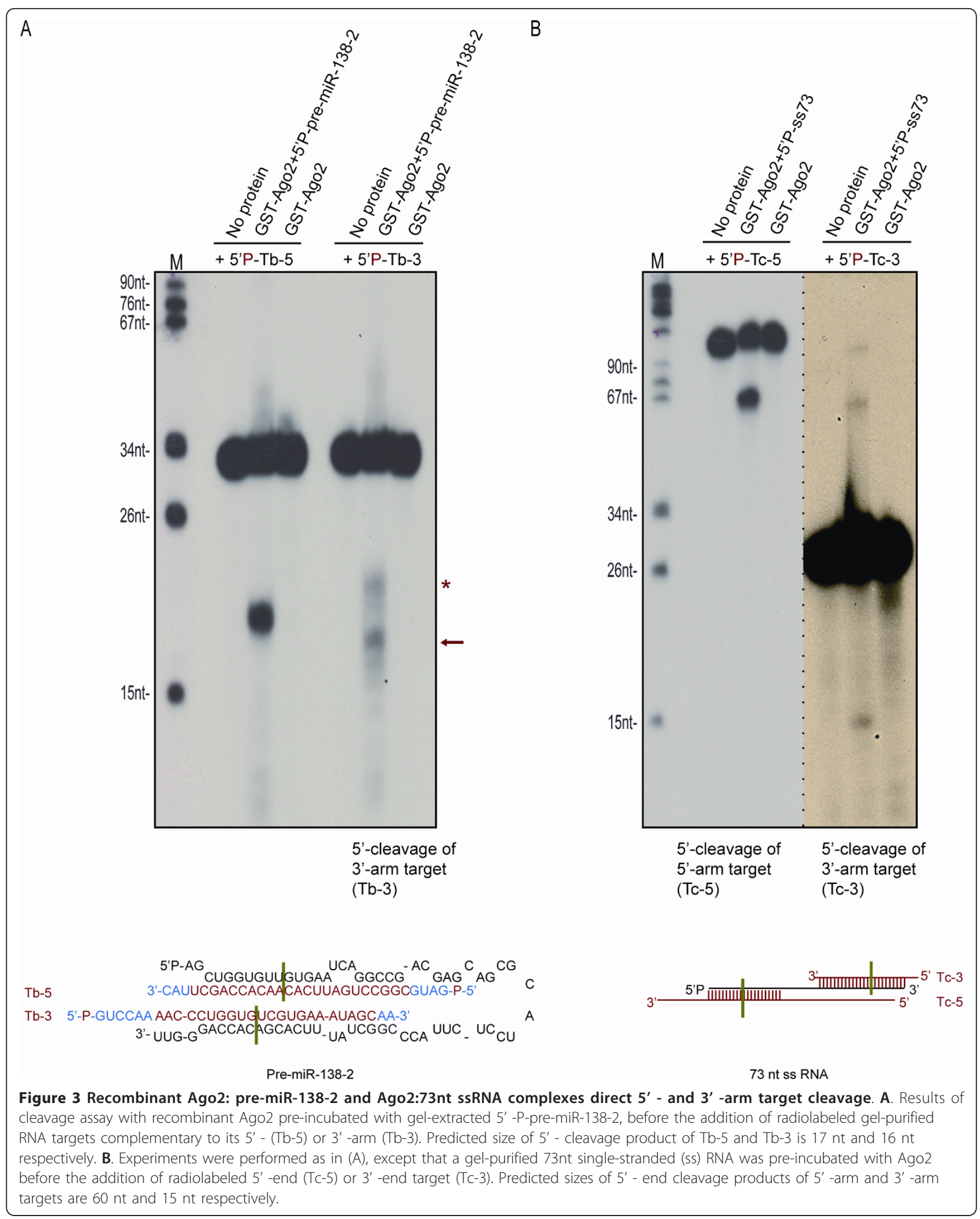


A

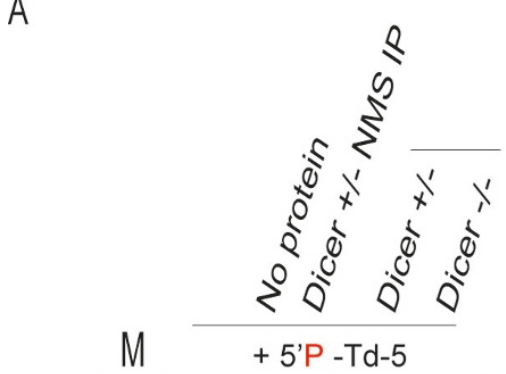

90nt-

$76 n t-$

67nt-

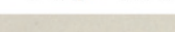

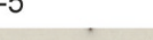

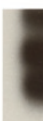
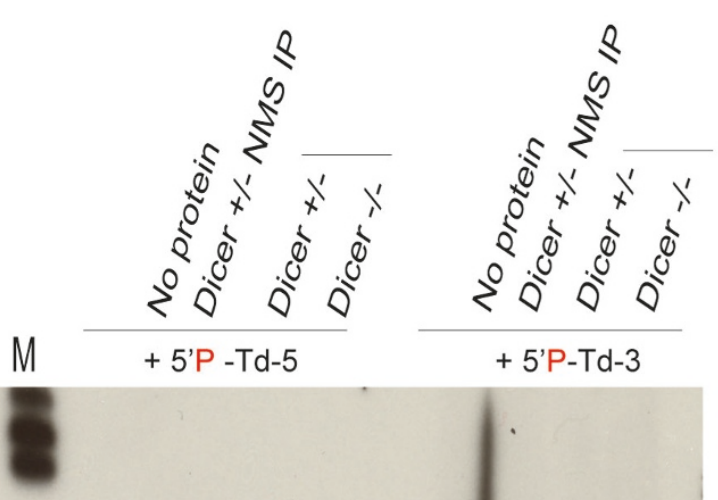

34nt-

26nt-
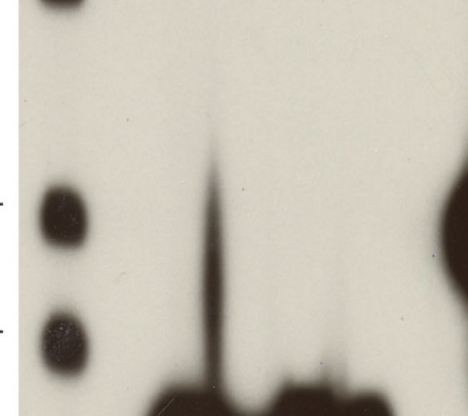

$15 n t-$

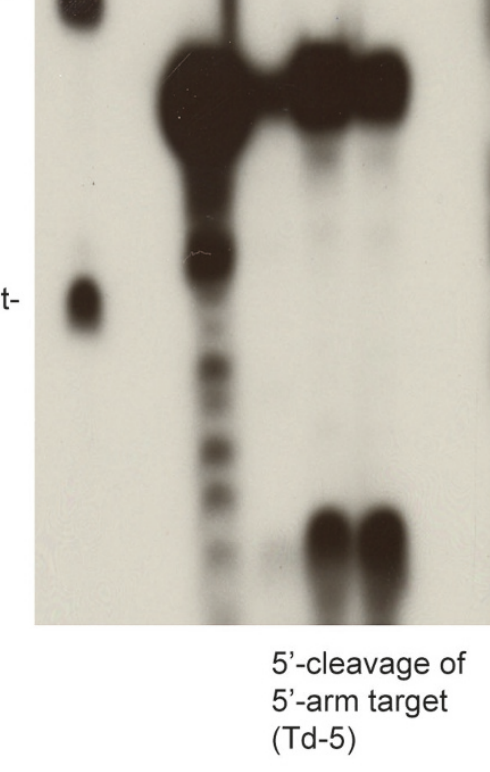

(Td-5)

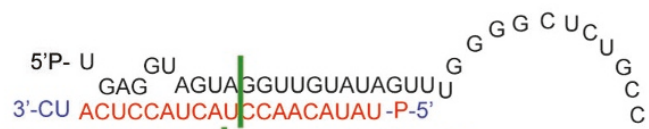

$$
\begin{aligned}
& \text { 3'-CU ACUCCAUCAU CCAACAUAU-P-5' } \\
& \text { Td-5 5'-P-UUCAUU GAAAGACAGY AGAUUGUAUAGCCG-3 } \\
& \text { Td-3 }
\end{aligned}
$$

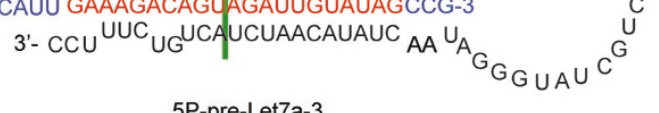

B
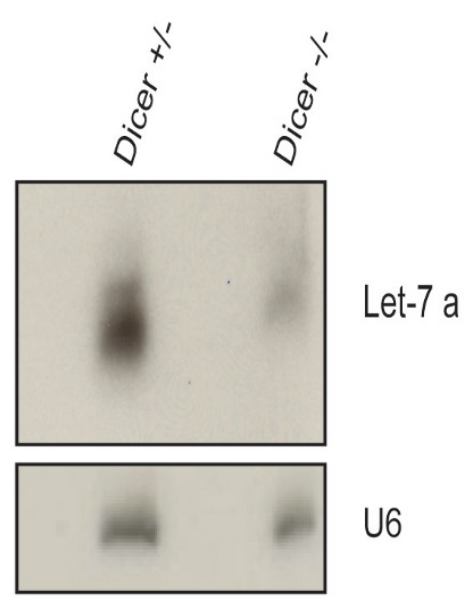

U6

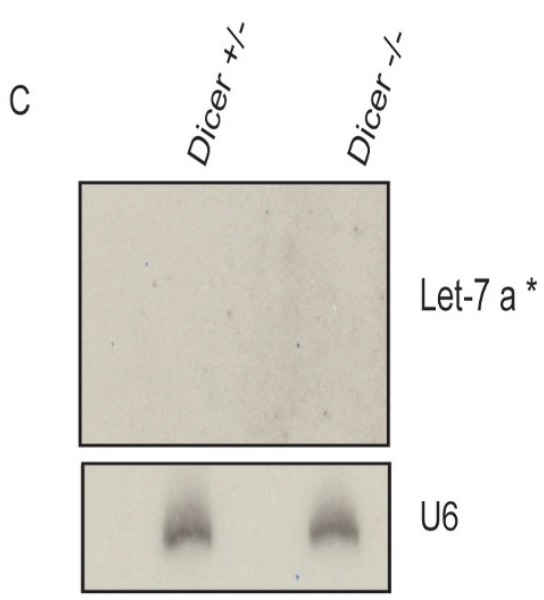

Figure 4 Endogenous pre-Let7a-3:Ago2 complexes guide 5'- and 3'- arm target cleavage in vitro. A. Endogenous pre-miRNPs were immunopurified using anti-Ago (2A8) antibody or from Dicer ${ }^{+/}$or Dicer $r^{-/}$mouse embryonic fibroblasts (MEFs). Non-immune mouse serum (NMS) was used as control. $30 \%$ of the total volume of agarose $\mathrm{G}$ beads from each immunoprecipitation (IP) was then incubated either with radiolabeled $5^{\prime}$-arm (Td-5) or with $3^{\prime}$-arm target (Td-3) and then treated with proteinase K. Predicted size of 5 -cleavage product of $5^{\prime}$-arm and $3^{\prime}$-arm target is 9 nt and 16 nt respectively. B and C. Expression of let-7a or let-7a * was detected by Northern blot of total RNA (20 $\mu \mathrm{g} / \mathrm{lane}$ ) isolated from Dicer ${ }^{+/}$or Dicer/- MEFs, using DNA antisense probes. For loading control, let-7a and let-7a* expression was normalized against the expression of U6 RNA. 
stem structure, with a perfect sense:antisense complementarity, resembling a 17 nt siRNA duplex. Our present and previous studies [31] indicate that pre-miRNAs with stem bulges are not processed by Ago2, but are used as guides for RISC activity in vitro. Our unexpected findings, also reported by Yoda et al [30], complicate in vitro dissection of RISC biogenesis and function.

In this study, we identified pre-miRNPs inactive against 3'-arm targets and this finding allowed us to demonstrate that Dicer and Ago2 are sufficient to process pre-miRNAs, unwind miRNA duplexes and load single-stranded miRNAs into Ago2. TRBP was dispensable for all three steps in vitro, arguing against an essential role for this protein in RISC reconstitution or miRNA strand selection but not excluding a regulatory function. Several studies have shown regulation of miRNA biogenesis by auxiliary factors (Reviewed in [36]). It has been previously shown that TRBP regulates pre-miRNA processing by Dicer [25-28]; furthermore, it has been proposed that TRBP is necessary to recruit Ago2 to the Dicer/substrate complexes [37]. Earlier reports suggested that TRBP in complex with Ago and Dicer is required for RISC loading reconstitution in vitro [32]. Our studies indicate that TRBP is not essential for this process.

Apart from clarifying the minimal factors required for mammalian RISC loading, our results suggest that premiRNAs bind to Ago2 via either their 5' - or 3'- end. Crystal structure studies have shown that the 3'- $[38,39]$ and the 5'- end [40] of miRNAs bind to Argonaute proteins and that there is a strong preference for $\mathrm{U}$ or $\mathrm{A}$ as a first 5 '- end base $[41,42]$. It is likely that pre-miRNAs are anchored within Ago 2 in a similar fashion. We tested four differently structured pre-miRNAs and the size of the 5 '-arm target product consistently corresponded to the expected cleavage across the nucleotide in position 10 from the 5 -end of the pre-miRNA. Most of the 3'-arm target products corresponded also to clea-

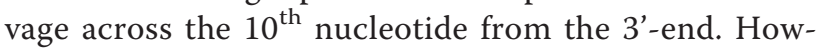
ever, additional products were detected suggesting imprecise cleavage, likely due to weak Ago2 binding to the 3'-unphosphorylated pre-miRNA end. It is unlikely that these RNA products represent degradation artifacts, as all the radiolabeled RNA oligos were gel-purified.

\section{Conclusions}

Our results indicate that Ago2 and Dicer are sufficient for processing and loading of miRNAs into RISC. Furthermore, our studies suggest that Ago2 binds primarily to the 5'-and alternatively, to the 3'-end of select pre-miRNAs. Results from our experiments using immunopurified pre-miRNPs from Dicer-null cells also support this alternative mode of endogenous premiRNA binding to Ago2. Endogenous pre-miRNPs accumulate in Dicer-deficient cells, likely because the secondary structure of pre-miRNAs and/or their binding by Ago 2 protects them from degradation; normally, Ago 2 containing pre-miRNPs are not abundant [31]. Since it is unlikely that miRNPs and pre-miRNPs exert redundant regulatory functions on gene expression, our in vitro findings should be cautiously interpreted, until the function of pre-miRNPs and biological significance of their RISC activity are elucidated.

\section{Methods}

\section{Expression and Purification of GST-Ago 2 and GST-TRBP}

Recombinant GST-Ago2 and GST-tagged human TRBP2 isoform a (NM_134323.1) were expressed using the baculovirus protein expression system and purified as described previously [31]. For protein purification, the baculovirus-infected $S f 9$ cell pellets were sonicated and recombinant protein was purified by Glutathione sepharose beads, washed and further purified by anion exchange chromatography. Silver nitrate gel analyses and pre-miRNA processing assays were performed with every protein batch to ensure purity of recombinant Ago 2 or TRBP and absence of Dicer (or Ago2 from TRBP preparations) or other associating proteins. Western Blot analyses with primary antibodies against Dicer (Novus NBP1-06520, 1:2000), 2 A8 ([33], 1:1000) and TRBP ([25], 1:1000) were also performed (Additional File 5).

\section{5'- labeling of RNAs}

RNA oligonucleotides were 5'-end radiolabeled with T4 polynucleotide kinase (NEB M0201S) as previously described $[43,44]$.

\section{Cleavage and Reconstitution Assays}

Cleavage assays were performed as previously described [31]. For reconstitution assays, $20 \mathrm{ng}$ recombinant GSTAgo2, GST-TRBP and/or Dicer (Ambion AM2212) were pre-incubated with $20 \mathrm{nM}$ of $5^{\prime}$-P-pre-miRNA at $37^{\circ} \mathrm{C}$ for $30 \mathrm{~min}$. $\sim 5 \mathrm{nmol}$ of gel extracted, 5'-radiolabeled RNA target was then added to the reactions, which were then incubated at $37^{\circ} \mathrm{C}$ for additional $60 \mathrm{~min}$. Cleavage products were then analyzed on $15 \%$ UREA PAGE. A 5' radiolabeled size marker (pBR322/MspI, NEB) was used for size control and radiolabeled cleavage products were detected by autoradiography. Nucleotide sequences of synthetic RNAs are listed in Additional File 6.

To analyze pre-miRNAs and their products in reconstitution assays, 5 '- ${ }^{32} \mathrm{P}$ - pre-miR-30a (20 nM) or $5^{\prime}-{ }^{32} \mathrm{P}$ pre-miR-24-1, was incubated with 20 ng GST-Ago2, GST-TRBP and/or Dicer at $37^{\circ} \mathrm{C}$ for $30 \mathrm{~min}$ before addition of cold RNA targets for $60 \mathrm{~min}$. 50 percent of the reaction volume was analyzed on 15\% UREA PAGE. The remaining 50 percent was mixed with native RNA 
loading buffer (6\% w/v Ficoll 400, 0.08\% bromophenol blue (w/v) and $4 \mathrm{mM}$ Tris pH7.5) and analyzed on 0.75 $\mathrm{mm} 15 \%$ native polyacrylamide (19:1), 0.5X TBE gel at $125 \mathrm{~V}$ (80-25 mA). Radiolabeled RNA products were detected by autoradiography.

\section{Dicer-null mouse embryonic fibroblasts (MEFs)}

The inducible Dicer-null MEFs were immortalized, cultured and induced as previously described [31].

\section{Immunoprecipitations and Northern blot analyses}

Dicer-null MEF lysates were incubated with $20 \mu \mathrm{L}$ of protein G agarose beads (Roche \#11 243233 001) prebound to $2 \mu \mathrm{L}$ of non-immune mouse serum or $20 \mu \mathrm{L}$ of anti-Ago 2A8 antibody [33] at $4^{\circ} \mathrm{C}$ overnight. Agarose beads were then washed five times in lysis buffer $(20$ $\mathrm{mM}$ Tris pH7.5, $200 \mathrm{mM} \mathrm{NaCl}, 2.5 \mathrm{mM} \mathrm{MgCl}_{2}$ and $0.5 \%$ Triton X-100) containing protease inhibitors (BMB \#11697498001). 10 percent of total protein G agarose volume was used for Western blot and 30 percent for cleavage assay.

Northern blot analyses of total RNA isolated from Dicer-null MEFs (miRVana, Ambion) were performed as previously described $[43,44] .5^{3}-{ }^{32} \mathrm{P}$ - let-7a or let- $7 \mathrm{a}^{*}$ DNA antisense probes were used to detect let-7a and let-7a* expression. For loading control, miRNA expression was normalized against the expression of U6, detected with a DNA antisense probe.

\section{Additional material}

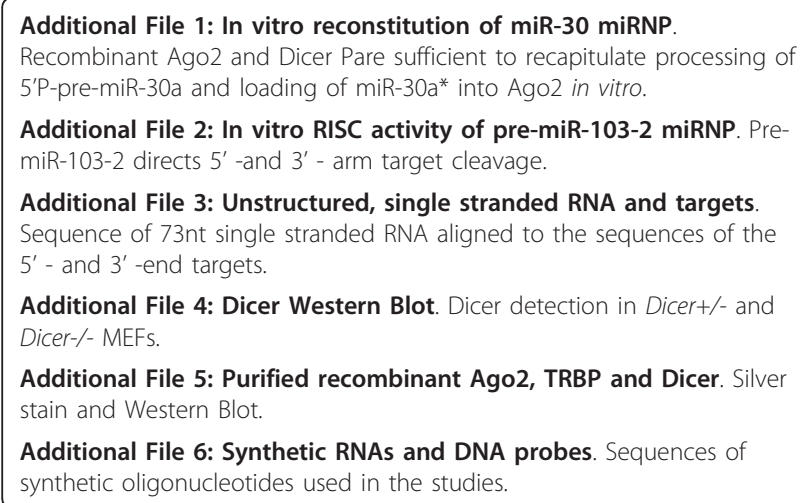

Additional File 5: Purified recombinant Ago2, TRBP and Dicer. Silver stain and Western Blot.

Additional File 6: Synthetic RNAs and DNA probes. Sequences of synthetic oligonucleotides used in the studies.

\begin{abstract}
Acknowledgements
The authors are grateful to Qinghua Liu for providing TRBP antibody. This work was supported by grants from National Institutes of Health/National Institute of Allergy and Infectious Diseases [K08Al063030 to M.K.]; the University of Pennsylvania [to M.K.] and the National Institutes of Health/ National Institute of Arthritis and Musculoskeletal and Skin Diseases [132AR007442-22 to B.G.G.]
\end{abstract}

\section{Author details}

'Department of Medicine, Division of Rheumatology, University of Pennsylvania School of Medicine, Philadelphia, PA, USA. '2Department of
Pathology, University of Pennsylvania School of Medicine, Philadelphia, PA, USA.

\section{Authors' contributions}

GST participated in the design of the study, carried out the in vitro assays and co-drafted the manuscript. BGG and DM carried out the purification of recombinant proteins. XL assisted GST in experimental work with Dicer-null cells. MK co-designed and supervised the study, compiled the figures and co-drafted the manuscript. All authors read and approved the final manuscript. The authors declare that they have no competing interests.

Received: 29 October 2010 Accepted: 29 April 2011

Published: 29 April 2011

\section{References}

1. Hammond SM, Boettcher S, Caudy AA, Kobayashi R, Hannon GJ: Argonaute2, a link between genetic and biochemical analyses of RNAi. Science 2001, 293(5532):1146-1150.

2. Hutvagner $G$, Zamore PD: A microRNA in a multiple-turnover RNAi enzyme complex. Science 2002, 297(5589):2056-2060.

3. Liu J, Carmell MA, Rivas FV, Marsden CG, Thomson JM, Song JJ, Hammond SM, Joshua-Tor L, Hannon GJ: Argonaute2 is the catalytic engine of mammalian RNAi. Science 2004, 305(5689):1437-1441.

4. Meister G, Landthaler M, Patkaniowska A, Dorsett Y, Teng G, Tuschl T: Human Argonaute2 mediates RNA cleavage targeted by miRNAs and siRNAs. Mol Cell 2004, 15(2):185-197.

5. Mourelatos Z, Dostie J, Paushkin S, Sharma A, Charroux B, Abel L, Rappsilber J, Mann M, Dreyfuss G: miRNPs: a novel class of ribonucleoproteins containing numerous microRNAs. Genes Dev 2002, 16(6):720-728.

6. Lewis $\mathrm{BP}$, Burge $\mathrm{CB}$, Bartel DP: Conserved seed pairing, often flanked by adenosines, indicates that thousands of human genes are microRNA targets. Cell 2005, 120(1):15-20.

7. Stefani G, Slack FJ: Small non-coding RNAs in animal development. Nat Rev Mol Cell Biol 2008, 9(3):219-230.

8. Kloosterman WP, Plasterk RH: The diverse functions of microRNAs in animal development and disease. Dev Cell 2006, 11(4):441-450.

9. Erson AE, Petty EM: miRNAs and cancer: New research developments and potential clinical applications. Cancer Biol Ther 2009, 8(24):2317-2322.

10. Huang ZP, Neppl RL, Wang DZ: MicroRNAs in cardiac remodeling and disease. J Cardiovasc Transl Res 2010, 3(3):212-218.

11. Mishra PK, Tyagi N, Kumar M, Tyagi SC: MicroRNAs as a therapeutic target for cardiovascular diseases. J Cell Mol Med 2009, 13(4):778-789.

12. Pauley KM, Cha S, Chan EK: MicroRNA in autoimmunity and autoimmune diseases. J Autoimmun 2009, 32(3-4):189-194.

13. Sonkoly E, Pivarcsi A: Advances in microRNAs: implications for immunity and inflammatory diseases. J Cell Mol Med 2009, 13(1):24-38.

14. Vinuesa CG, Rigby RJ, Yu D: Logic and extent of miRNA-mediated control of autoimmune gene expression. Int Rev Immunol 2009, 28(34):112-138.

15. Landthaler M, Yalcin A, Tuschl T: The human DiGeorge syndrome critical region gene 8 and Its $D$. melanogaster homolog are required for miRNA biogenesis. Curr Biol 2004, 14(23):2162-2167.

16. Denli AM, Tops BB, Plasterk RH, Ketting RF, Hannon GJ: Processing of primary microRNAs by the Microprocessor complex. Nature 2004, 432(7014):231-235

17. Han J, Lee Y, Yeom KH, Kim YK, Jin H, Kim VN: The Drosha-DGCR8 complex in primary microRNA processing. Genes Dev 2004, 18(24):3016-3027.

18. Kim VN, Han J, Siomi MC: Biogenesis of small RNAs in animals. Nat Rev Mol Cell Biol 2009, 10(2):126-139.

19. Bohnsack MT, Czaplinski K, Gorlich D: Exportin 5 is a RanGTP-dependent dsRNA-binding protein that mediates nuclear export of pre-miRNAs. Rna 2004, 10(2):185-191.

20. Kim VN: MicroRNA precursors in motion: exportin-5 mediates their nuclear export. Trends Cell Biol 2004, 14(4):156-159.

21. Lund E, Guttinger S, Calado A, Dahlberg JE, Kutay U: Nuclear export of microRNA precursors. Science 2004, 303(5654):95-98.

22. Yi R, Qin Y, Macara IG, Cullen BR: Exportin-5 mediates the nuclear export of pre-microRNAs and short hairpin RNAs. Genes Dev 2003, 17(24):3011-3016. 
23. Bernstein E, Caudy AA, Hammond SM, Hannon GJ: Role for a bidentate ribonuclease in the initiation step of RNA interference. Nature 2001, 409(6818):363-366.

24. Nicholson RH, Nicholson AW: Molecular characterization of a mouse cDNA encoding Dicer, a ribonuclease III ortholog involved in RNA interference. Mamm Genome 2002, 13(2):67-73.

25. Paroo Z, Ye X, Chen S, Liu Q: Phosphorylation of the human microRNAgenerating complex mediates MAPK/Erk signaling. Cell 2009, 139(1):112-122.

26. Melo SA, Ropero S, Moutinho C, Aaltonen LA, Yamamoto H, Calin GA, Rossi S, Fernandez AF, Carneiro F, Oliveira C, et al: A TARBP2 mutation in human cancer impairs microRNA processing and DICER1 function. Nat Genet 2009, 41(3):365-370.

27. Kok KH, Ng MH, Ching YP, Jin DY: Human TRBP and PACT directly interact with each other and associate with dicer to facilitate the production of small interfering RNA. J Biol Chem 2007, 282(24):17649-17657.

28. Haase AD, Jaskiewicz L, Zhang H, Laine S, Sack R, Gatignol A, Filipowicz W: TRBP, a regulator of cellular PKR and HIV-1 virus expression, interacts with Dicer and functions in RNA silencing. EMBO Rep 2005, 6(10):961-967.

29. Wang B, Li S, Qi HH, Chowdhury D, Shi Y, Novina CD: Distinct passenger strand and mRNA cleavage activities of human Argonaute proteins. Nat Struct Mol Biol 2009, 16(12):1259-1266

30. Yoda M, Kawamata T, Paroo Z, Ye X, Iwasaki S, Liu Q, Tomari Y: ATPdependent human RISC assembly pathways. Nat Struct Mol Biol 2010, 17(1):17-23.

31. Tan GS, Garchow BG, Liu X, Yeung J, Morris JPt, Cuellar TL, McManus MT, Kiriakidou M: Expanded RNA-binding activities of mammalian Argonaute 2. Nucleic Acids Res 2009, 37(22):7533-7545.

32. MacRae IJ, Ma E, Zhou M, Robinson CV, Doudna JA: In vitro reconstitution of the human RISC-loading complex. Proc Natl Acad Sci USA 2008, 105(2):512-517.

33. Nelson PT, De Planell-Saguer M, Lamprinaki S, Kiriakidou M, Zhang P, O'Doherty U, Mourelatos Z: A novel monoclonal antibody against human Argonaute proteins reveals unexpected characteristics of miRNAs in human blood cells. Rna 2007, 13(10):1787-1792.

34. Cheloufi S, Dos Santos CO, Chong MM, Hannon GJ: A dicer-independent miRNA biogenesis pathway that requires Ago catalysis. Nature 2010, 465(7298):584-589.

35. Cifuentes D, Xue H, Taylor DW, Patnode H, Mishima Y, Cheloufi S, Ma E, Mane S, Hannon GJ, Lawson ND, Wolfe SA, Giraldez AJ: A novel miRNA processing pathway independent of Dicer requires Argonaute2 catalytic activity. Science 2010, 328(5986):1694-1698.

36. Krol J, Loedige I, Filipowicz W: The widespread regulation of microRNA biogenesis, function and decay. Nat Rev Genet 2010, 11(9):597-610.

37. Gregory RI, Chendrimada TP, Cooch N, Shiekhattar R: Human RISC couples microRNA biogenesis and posttranscriptional gene silencing. Cell 2005, 123(4):631-640.

38. Ma JB, Ye K, Patel DJ: Structural basis for overhang-specific small interfering RNA recognition by the PAZ domain. Nature 2004, 429(6989):318-322

39. Song JJ, Liu J, Tolia NH, Schneiderman J, Smith SK, Martienssen RA, Hannon GJ, Joshua-Tor L: The crystal structure of the Argonaute2 PAZ domain reveals an RNA binding motif in RNAi effector complexes. Nat Struct Biol 2003, 10(12):1026-1032.

40. Ma JB, Yuan YR, Meister G, Pei Y, Tuschl T, Patel DJ: Structural basis for 5' end-specific recognition of guide RNA by the A. fulgidus Piwi protein. Nature 2005, 434(7033):666-670.

41. Faehnle CR, Joshua-Tor L: Argonaute MID domain takes centre stage. EMBO Rep 2010, 11(8):564-565.

42. Frank F, Sonenberg N, Nagar B: Structural basis for $5^{\prime}$-nucleotide basespecific recognition of guide RNA by human AGO2. Nature 2010, 465(7299):818-822.

43. Kiriakidou M, Nelson P, Lamprinaki S, Sharma A, Mourelatos Z: Detection of microRNAs and assays to monitor microRNA activities in vivo and in vitro. Methods Mol Biol 2005, 309:295-310.

44. Maniataki E, De Planell Saguer MD, Mourelatos Z: Immunoprecipitation of microRNPs and directional cloning of microRNAs. Methods Mol Biol 2005 309:283-294.

doi:10.1186/1471-2199-12-19

Cite this article as: Tan et al: Clarifying mammalian RISC assembly in vitro. BMC Molecular Biology 2011 12:19.

\section{Submit your next manuscript to BioMed Central and take full advantage of:}

- Convenient online submission

- Thorough peer review

- No space constraints or color figure charges

- Immediate publication on acceptance

- Inclusion in PubMed, CAS, Scopus and Google Scholar

- Research which is freely available for redistribution

Submit your manuscript at www.biomedcentral.com/submit
Biomed Central 